\title{
Treatment of Refractory Postdural Puncture Headache after Intrathecal Drug Delivery System Implantation with Epidural Blood Patch Procedures: A 20-Year Experience
}

\author{
Markus A. Bendel, ${ }^{1}$ Susan M. Moeschler, ${ }^{1}$ Wenchun Qu, ${ }^{1}$ Eugerie Hanley, ${ }^{2}$ \\ Stephanie A. Neuman, ${ }^{3}$ Jason S. Eldrige, ${ }^{1}$ and Bryan C. Hoelzer ${ }^{1}$ \\ ${ }^{1}$ Mayo Clinic, Rochester, MN 55905, USA \\ ${ }^{2}$ Ozark Orthopaedics, Fayetteville, AR 72703, USA \\ ${ }^{3}$ Gundersen-Lutheran Health System, La Crosse, WI 54601, USA \\ Correspondence should be addressed to Markus A. Bendel; bendel.markus@mayo.edu
}

Received 26 May 2016; Accepted 4 July 2016

Academic Editor: Hartmut Göbel

Copyright ( $) 2016$ Markus A. Bendel et al. This is an open access article distributed under the Creative Commons Attribution License, which permits unrestricted use, distribution, and reproduction in any medium, provided the original work is properly cited.

\begin{abstract}
A recent publication reported the incidence of postdural puncture headache (PDPH) in conjunction with intrathecal drug delivery system (IDDS) implantation to be nearly 23 percent. Many patients responded to conservative measures but a percentage needed invasive treatment with an epidural blood patch (EBP). There is limited data to describe the technical details, success rates, and complications associated with EBP in this population. This study aims to provide a retrospective report of EBP for patients suffering from PDPH related to IDDS implantation. A chart review established a cohort of patients that required EBP in relation to a PDPH after IDDS implantation. This cohort was evaluated for demographic data as well as details of the EBP including technical procedural data, success rates, and complications. All patients received a trial of conservative therapy. Standard sterile technique and skin preparation were utilized with no infectious complications. The EBP was placed below the level of the IDDS catheter in $94 \%$ of procedures. Fluoroscopy was utilized in each case. The mean EBP volume was $18.6 \mathrm{cc}$ and median time of EBP was day 7 after implant. There were no complications associated with EBP. EBP appears to be an effective intervention in this subset of PDPH patients.
\end{abstract}

\section{Introduction}

Intrathecal drug delivery system (IDDS) implantation can be an efficacious tool in managing patients with spasticity, chronic malignant or nonmalignant pain [1-9]. Many advances in our understanding of intrathecal medication administration and steady improvements in technology have helped to shape the best practices associated with device implantation and management [10]. However, this intervention is not without complication. Hematoma formation, neurologic injury, system failure, infection, catheter-associated granuloma formation, opioid induced hyperalgesia, continuous cerebrospinal fluid leak, seroma formation, and postdural puncture headache $(\mathrm{PDPH})$ are known complications of IDDS placement [11-17].
A recently published retrospective review examining the incidence of PDPH in conjunction with IDDS implantation found symptoms in nearly 23 percent of patients [11]. The majority of these patients were managed conservatively with standard of care noninvasive measures including bedrest, intravenous hydration, caffeine supplementation, and analgesic medication administration [18, 19]. However, some patients continued to suffer severe symptoms of PDPH warranting an interventional management approach.

Epidural blood patch (EBP) is a well-described technique used to provide relief to patients suffering from resistant or severe PDPH. There is evidence to support its efficacy in relieving postdural puncture headache [20-23]. However, debate still remains regarding the necessity, effectiveness, timing, and exact technique of EBP [24, 25]. EBP 
carries a small risk of complications including unintended dural puncture, subarachnoid spread, hematoma formation, increased intracranial pressure, and neurologic injury [2629]. However, the combination of the known risks of EBP and patients who have long-term intrathecal catheters in situ prompts new consideration. There are case reports describing the application of EBP or fibrin glue patch in patients with IDDS implants in the setting of continuous cerebrospinal fluid leak [30-33]. There is a scarcity of published material describing the outcomes, technical details, and complications associated with epidural blood patch in the PDPH population with IDDS in place. This provides a difficult landscape from which to ascertain the best practice in managing this small but important subset of patients with PDPH.

\section{Objective}

The objective of the current study was to provide a detailed review of management of refractory postdural puncture headache in patients with implanted IDDS who required epidural blood patch procedures over a 20-year time frame at a single academic tertiary care medical center (Mayo Clinic, Rochester, MN).

\section{Methods}

Following appropriate institutional review board (IRB) approval, we performed a retrospective cohort study based upon a 20-year chart review of clinical experience at a large academic medical center (Mayo Clinic, Rochester, MN). Patients who underwent placement of an IDDS during the period between June 1, 1989, and May 31, 2009, were included. The Mayo Clinic medical data retrieval service identified all patients who underwent implantation of an IDDS in this time frame with the use of ICD-9 codes. As described in the prior publication [11], a subset of patients who had symptoms consistent with PDPH was identified by examining all inpatient and outpatient clinical notes for a three-week period following IDDS implantation. This subset was examined for patients that proceeded to invasive intervention in the form of epidural blood patch. This formed the cohort.

The following data points were harvested from each medical record of the cohort: age at time of IDDS implantation, gender, indication for IDDS, date of IDDS implantation, methods of conservative PDPH management, IDDS catheter entry level, epidural blood patch level, epidural blood patch volume, interval between IDDS implantation and epidural blood patch, and preepidural blood patch skin preparation method. Improvement in headache symptoms after blood patch was recorded. Finally, each record was carefully screened for immediate and delayed post-EBP complications.

\section{Results}

The medical data retrieval service returned 319 surgical reports on 285 different patients over the specified time frame. As previously reported, chart review uncovered postdural puncture headache symptoms in 73 patients $(22.9 \%)$
[11]. Of the patients with PDPH, 15 patients had symptoms refractory to conservative treatment and required 17 epidural blood patch procedures. The complete results are summarized in Table 1.

4.1. Conservative Treatments. All patients received a trial of at least one conservative measure. The conservative measures and their incidence of use in this cohort are as follows: bedrest (100\%), intravenous hydration (67\%), caffeine (67\%), acetaminophen (47\%), and antiemetic therapy (27\%). See Table 2.

4.2. Infectious Precautions. All epidural blood patches were performed under standard sterile technique. Three cases did not have skin preparation explicitly documented (20\%). All others were completed with chlorhexidine $(60 \%)$, betadine $(13 \%)$, or both $(7 \%)$. One patient received prophylactic intravenous antibiotics due to proceduralist preference.

4.3. Image Guidance. All of the procedures were performed with fluoroscopic guidance to ensure proper needle positioning.

4.4. EBP Level. The blood patch was performed below the level of the IDDS catheter insertion in $94 \%$ of cases. See Table 3 .

4.5. EBP Blood Volume. One procedure did not have volume administered explicitly recorded in the medical chart. The mean volume for the remaining procedures was $18.6 \mathrm{~mL}$. The volume administered was determined by the development of patient reported paresthesias or a maximum volume of $20 \mathrm{~mL}$.

4.6. EBP Timing. The initial blood patch was placed on median postimplantation day number 7 . The range of initial blood patch timing ranged from 3 to 36 days after IDDS implantation. Two patients required repeat blood patch application-these occurred on days 15 and 28 after implant which were 3 and 8 days after the initial EBP, respectively. See Table 4.

4.7. PDPH Symptom Resolution. All patients reviewed achieved relief of the PDPH symptoms. First-time blood patch success rate was 10/15 (67\%). Two patients required repeat blood patches that were both successful (13\%). Three patients $(20 \%)$ were found to have persistent cerebrospinal fluid leak that required reoperation with purse-string suture placement and/or surgical fibrin glue placement at the IDDS catheter entrance site.

4.8. Complications. We found no complications directly related to performing an epidural blood patch in this cohort.

\section{Discussion}

The goal of this study was to address the relative paucity of published technical data in placing an epidural blood 


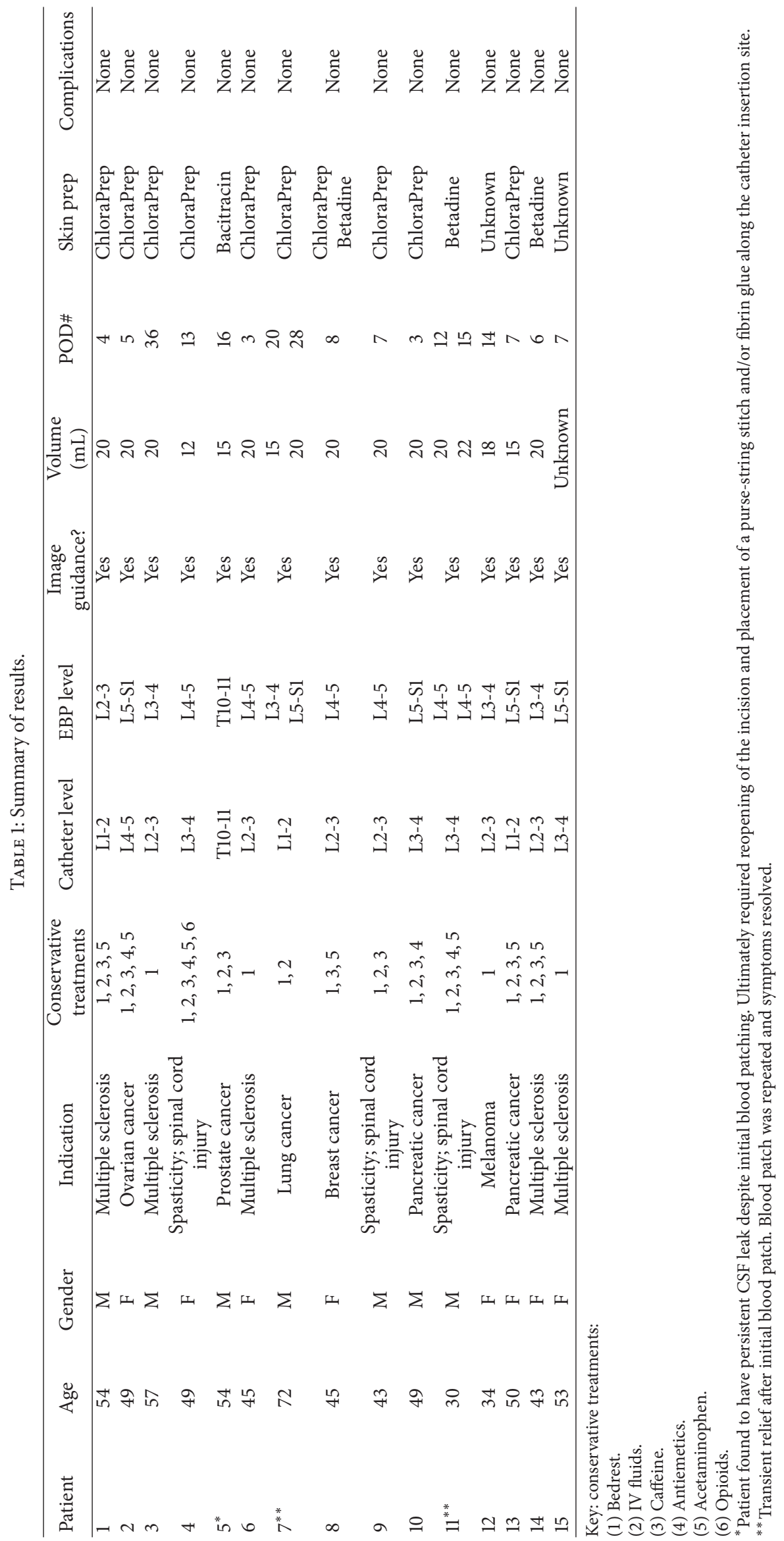


TABLE 2: Incidence of conservative treatments.

\begin{tabular}{lc}
\hline Number of conservative treatments employed & Incidence \\
\hline 1 & $27 \%(4 / 15)$ \\
2 & $7 \%(1 / 15)$ \\
3 & $20 \%(3 / 15)$ \\
$4+$ & $47 \%(7 / 15)$ \\
\hline
\end{tabular}

TABLE 3: Location of epidural blood patch.

\begin{tabular}{lc}
\hline Location of EBP relative to IDDS catheter & Incidence \\
\hline At IDDS insertion site & $6 \%(1 / 17)$ \\
1 level below & $47 \%(8 / 17)$ \\
2 levels below & $35 \%(6 / 17)$ \\
3 or more levels below & $12 \%(2 / 17)$ \\
\hline
\end{tabular}

TABLE 4: Timing of epidural blood patch after IDDS implant.

\begin{tabular}{ll}
\hline Time of EBP after IDDS implant & Incidence \\
\hline $1-7$ days & $53 \%(8 / 15)$ \\
$8-14$ days & $27 \%(4 / 15)$ \\
$15+$ days & $20 \%(3 / 15)$ \\
\hline
\end{tabular}

patch in patients with refractory PDPH in the setting of a recently placed IDDS. As previously reported, the incidence of PDPH in this population is likely higher than initially believed [11]. Conservative measures are effective in relieving the underlying symptoms in the majority of cases. However, a portion of this population continues to have significant amount of PDPH symptomology that is deleterious to their quality of life. This series suggests that epidural blood patches can be performed in this population with a reasonable margin of safety and efficacy. We found no increased incidence of complications. There are, however, case reports in the literature of complications utilizing this technique in this population [34]. Given the minimal amount of data in the literature relating EBP, PDPH, and IDDS, it is difficult to estimate the true incidence of complications in this population compared with other PDPH and EBP populations.

There do not appear to be any technical nuances specific to performing an EBP in a patient with IDDS, other than avoiding direct trauma to the IDDS catheter in situ by utilizing fluoroscopy. This series would suggest that standard sterile technique with betadine or chlorhexidine skin preparation is satisfactory for infectious prophylaxis. Only one patient received intravenous prophylactic antibiotics and there were no infections observed in our dataset. It has been an effective practice to place the EBP from 1 to 4 interspaces below the site of the IDDS catheter. The volume administered varied according to development of patient reported paresthesias, but often the patient received the goal volume of twenty milliliters. The practice of performing an epidural blood patch has evolved to include higher volumes of administered blood due to data highlighting the importance of pressure paresthesia $[28,29]$. Therefore, twenty milliliters may not have been an optimal volume in those patients who did not develop a pressure paresthesia. Despite this, a large majority of patients (80\%) did report relief from one or two blood patches.

Epidural blood patching appears to be an effective intervention for patients who are afflicted with PDPH related to IDDS implantation. A majority of the patients obtained lasting relief after application of a single blood patch, while a small subset needed a second blood patch. This is consistent with success rates of EBP in other patient populations [22,23]. Of the patients who did not respond initially, it appears reasonable to proceed with repeat EBP or epidural fibrin glue administration. On rare occasions, patients may require operative intervention with dural repair or revision.

Limitations. This study has recognizable limitations. Although our dataset spans a time frame of 20 years, it includes only 15 patients in our cohort. It is difficult to extrapolate to an entire population based on this sample size. Secondly, a retrospective chart review study design is not sufficient to establish any cause-effect relationship, but it would be very difficult to construct a randomized, prospective trial to assess the effectiveness of this intervention. Lastly, this dataset includes a compilation of several proceduralists with varying preferences in their procedural technique. It is impossible to control for individual preference retrospectively and this makes the interpretation of the results more difficult.

\section{Conclusions}

In conclusion, this study has evaluated the largest known-todate cohort of patients with PDPH after IDDS implant and provided a technical report on how these patients have been managed with EBP. EBP appears to be an effective intervention in this subset of PDPH patients and no complications were observed utilizing the techniques as outlined above. Repeat blood patches or epidural fibrin glue administration may be helpful for patients who do not respond to initial EBP.

\section{Competing Interests}

The authors have no relevant competing interests to disclose.

\section{References}

[1] M. Wallace and T. L. Yaksh, "Long-term spinal analgesic delivery: a review of the preclinical and clinical literature," Regional Anesthesia and Pain Medicine, vol. 25, no. 2, pp. 117$157,2000$.

[2] S. M. Hayek, T. R. Deer, J. E. Pope, S. J. Panchal, and V. Patel, "Intrathecal therapy for cancer and non-cancer pain," Pain Physician, vol. 14, no. 3, pp. 219-248, 2011.

[3] P. Dahm, P. Nitescu, L. Appelgren, and I. Curelaru, "Efficacy and technical complications of long-term continuous intraspinal infusions of opioid and/or bupivacaine in refractory nonmalignant pain: a comparison between the epidural and the intrathecal approach with externalized or implanted catheters and infusion pumps," Clinical Journal of Pain, vol. 14, no. 1, pp. 4-16, 1998. 
[4] W. Raffaeli, D. Righetti, A. Caminiti et al., "Implantable intrathecal pumps for the treatment of noncancer chronic pain in elderly population: drug dose and clinical efficacy," Neuromodulation, vol. 11, no. 1, pp. 33-39, 2008.

[5] C.-P. Lin, W.-Y. Lin, F.-S. Lin, Y.-S. Lee, C.-S. Jeng, and W.-Z. Sun, "Efficacy of intrathecal drug delivery system for refractory cancer pain patients: a single tertiary medical center experience," Journal of the Formosan Medical Association, vol. 111, no. 5, pp. 253-257, 2012.

[6] K. Kumar, M. Kelly, and T. Pirlot, "Continuous intrathecal morphine treatment for chronic pain of nonmalignant etiology: long-term benefits and efficacy," Surgical Neurology, vol. 55, no. 2, pp. 79-88, 2001.

[7] T. R. Deer, H. S. Smith, A. Burton et al., "Comprehensive consensus based guidelines on intrathecal drug delivery systems in the treatment of pain caused by cancer pain," Pain Physician, vol. 14, no. 3, pp. E283-E312, 2011.

[8] H. S. Smith, T. R. Deer, P. S. Staats, V. Singh, N. Sehgal, and H. Cordner, "Intrathecal drug delivery," Pain Physician, vol. 11, no. 2, supplement, pp. S89-S104, 2008.

[9] S. N. Mathur, S. K. Chu, Z. McCormick, G. C. Chang Chien, and C. M. Marciniak, "Long-term intrathecal baclofen: outcomes after more than 10 years of treatment," $P M$ and $R$, vol. 6, no. 6, pp. 506-513.e1, 2014.

[10] T. R. Deer, J. Prager, R. Levy et al., "Polyanalgesic Consensus Conference 2012: recommendations for the management of pain by intrathecal (intraspinal) drug delivery: report of an interdisciplinary expert panel," Neuromodulation, vol. 15, no. 5, pp. 436-466, 2012.

[11] S. A. Neuman, J. S. Eldrige, W. Qu, E. D. Freeman, and B. C. Hoelzer, "Post dural puncture headache following intrathecal drug delivery system placement," Pain Physician, vol. 16, no. 2, pp. 101-107, 2013.

[12] B. J. P. Crul, “Technical complications during long-term subarachnoid or epidural administration of morphine in terminally 111 cancer patients: a review of 140 cases," Regional Anesthesia, vol. 16, no. 4, pp. 209-213, 1991.

[13] K. A. Follett and C. P. Naumann, "A prospective study of catheter-related complications of intrathecal drug delivery systems," Journal of Pain and Symptom Management, vol. 19, no. 3, pp. 209-215, 2000.

[14] S. Kamran and B. D. Wright, "Complications of intrathecal drug delivery systems," Neuromodulation, vol. 4, no. 3, pp. 111-115, 2001.

[15] R. J. Coffey, M. L. Owens, S. K. Broste et al., "Mortality associated with implantation and management of intrathecal opioid drug infusion systems to treat noncancer pain," Anesthesiology, vol. 111, no. 4, pp. 881-891, 2009.

[16] P. Gulur, G. Bhatia, M. E. Lau, and K. M. Koury, "Intrathecal Drug Delivery (ITDD) systems for cancer pain," F1000Research, vol. 2, p. 96, 2013.

[17] J. M. Hitt and O. A. de Leon-Casasola, "Complications of intrathecal drug delivery systems," Techniques in Regional Anesthesia and Pain Management, vol. 15, no. 4, pp. 162-166, 2011.

[18] X. Basurto Ona, L. Martínez García, I. Solà, and X. Bonfill Cosp, "Drug therapy for treating post-dural puncture headache," The Cochrane Database of Systematic Reviews, no. 8, Article ID CD007887, 2011.

[19] I. Arevalo-Rodriguez, A. Ciapponi, L. Munoz, M. Roqué i Figuls, and X. Bonfill Cosp, "Posture and fluids for preventing post-dural puncture headache," The Cochrane Database of Systematic Reviews, no. 7, Article ID CD009199, 2013.

[20] F. van Kooten, R. Oedit, S. L. M. Bakker, and D. W. J. Dippel, "Epidural blood patch in post dural puncture headache: a randomised, observer-blind, controlled clinical trial," Journal of Neurology, Neurosurgery and Psychiatry, vol. 79, no. 5, pp. 553558, 2008.

[21] V. Safa-Tisseront, F. Thormann, P. Malassiné et al., "Effectiveness of epidural blood patch in the management of post-dural puncture headache," Anesthesiology, vol. 95, no. 2, pp. 334-339, 2001.

[22] S. Banks, M. Paech, and L. Gurrin, "An audit of epidural blood patch after accidental dural puncture with a Tuohy needle in obstetric patients," International Journal of Obstetric Anesthesia, vol. 10, no. 3, pp. 172-176, 2001.

[23] P. C. Stride and G. M. Cooper, "Dural taps revisited. A 20-year survey from Birmingham maternity hospital," Anaesthesia, vol. 48, no. 3, pp. 247-255, 1993.

[24] S. Malhotra, "All patients with a postdural puncture headache should receive an epidural blood patch," International Journal of Obstetric Anesthesia, vol. 23, no. 2, pp. 168-170, 2014.

[25] O. M. W. M. Rucklidge, "All patients with a postdural puncture headache should receive an epidural blood patch," International Journal of Obstetric Anesthesia, vol. 23, no. 2, pp. 171-174, 2014.

[26] P. Kalina, P. Craigo, and T. Weingarten, "Intrathecal injection of epidural blood patch: a case report and review of the literature," Emergency Radiology, vol. 11, no. 1, pp. 56-59, 2004.

[27] C. A. Riley and J. E. Spiegel, "Complications following large-volume epidural blood patches for postdural puncture headache. Lumbar subdural hematoma and arachnoiditis: initial cause or final effect?" Journal of Clinical Anesthesia, vol. 21, no. 5, pp. 355-359, 2009.

[28] G. Zada, P. Pezeshkian, and S. Giannotta, "Spontaneous intracranial hypotension and immediate improvement following epidural blood patch placement demonstrated by intracranial pressure monitoring. Case report," Journal of Neurosurgery, vol. 106, no. 6, pp. 1089-1090, 2007.

[29] C. Dubost, A. Le Gouez, P. J. Zetlaoui, D. Benhamou, F. J. Mercier, and T. Geeraerts, "Increase in optic nerve sheath diameter induced by epidural blood patch: a preliminary report," British Journal of Anaesthesia, vol. 107, no. 4, pp. 627-630, 2011.

[30] E. Dominguez, O. Latif, D. Rozen et al., "Subdural blood patch for the treatment of persistent CSF leak after permanent intrathecal catheter implantation: a report of two cases," Pain Practice, vol. 1, no. 4, pp. 344-353, 2001.

[31] E. D. Freeman, B. C. Hoelzer, J. S. Eldrige, and S. M. Moeschler, "Fibrin glue to treat spinal fluid leaks associated with intrathecal drug systems," Pain Practice, vol. 14, no. 6, pp. 570-576, 2014.

[32] P. A. J. Hardy, "Extradural blood patch of a cerebrospinal fluid cutaneous fistula in the presence of an intrathecal drug delivery system," Anesthesiology, vol. 81, no. 5, pp. 1299-1300, 1994.

[33] P. K. Singh, R. Jain, S. Mishra, S. Kumar, S. Bhatnagar, and S. Deo, "Management of pericatheter cerebrospinal fluid leak after intrathecal implantation of a drug delivery system," American Journal of Hospice and Palliative Medicine, vol. 25, no. 3, pp. 237239, 2008.

[34] E. C. Hustak, M. P. Engle, A. Viswanathan, and D. Koyyalagunta, "Lumbar subarachnoid hematoma following an epidural blood patch for meningeal puncture headache related to the implantation of an intrathecal drug delivery system," Pain Physician, vol. 17, no. 3, pp. E405-E411, 2014. 


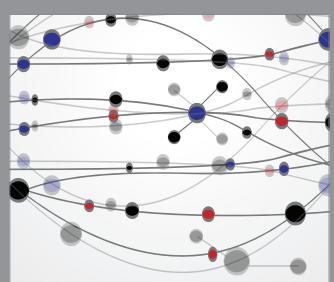

The Scientific World Journal
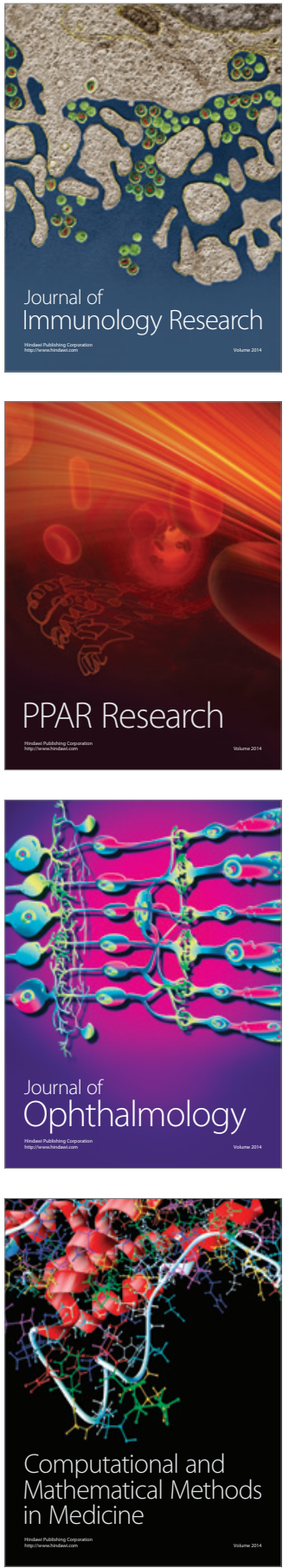

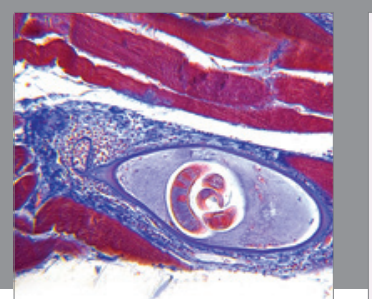

Gastroenterology Research and Practice

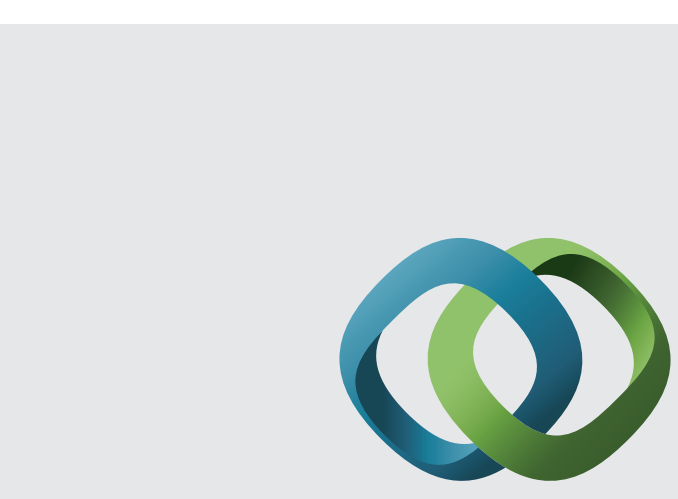

\section{Hindawi}

Submit your manuscripts at

http://www.hindawi.com
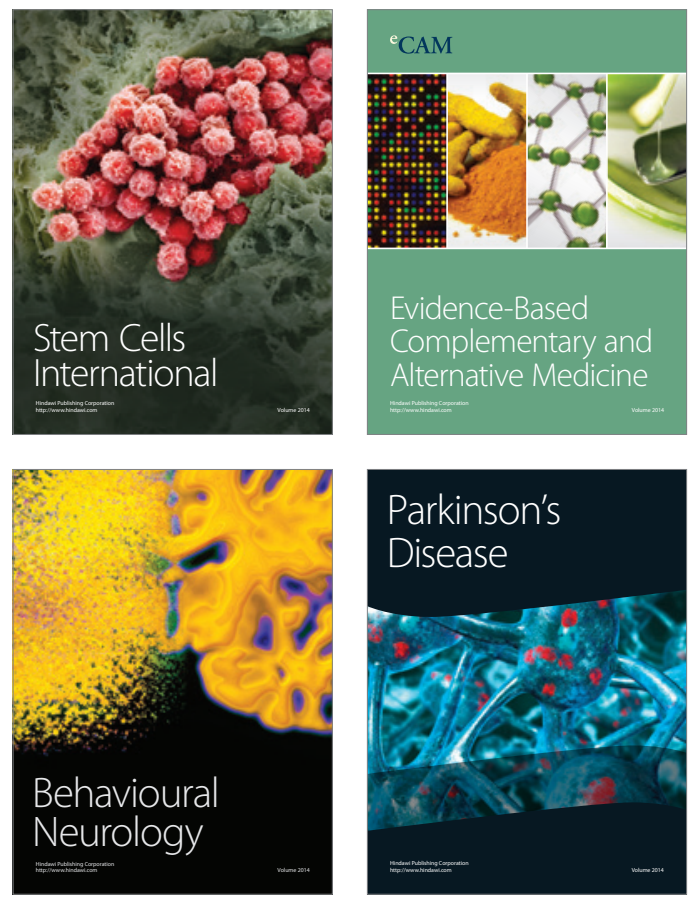
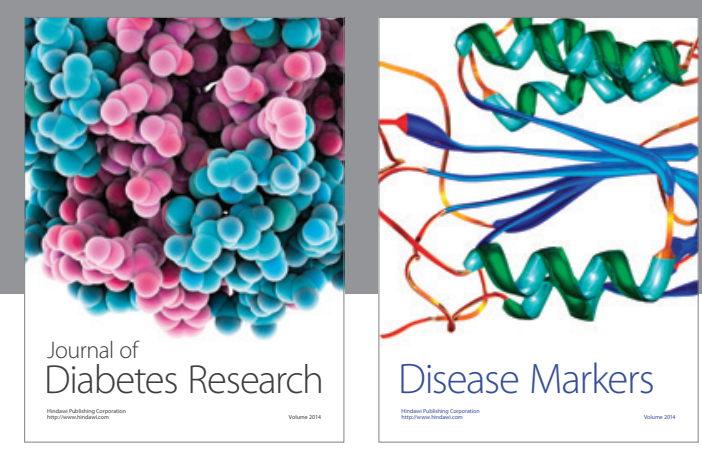

Disease Markers
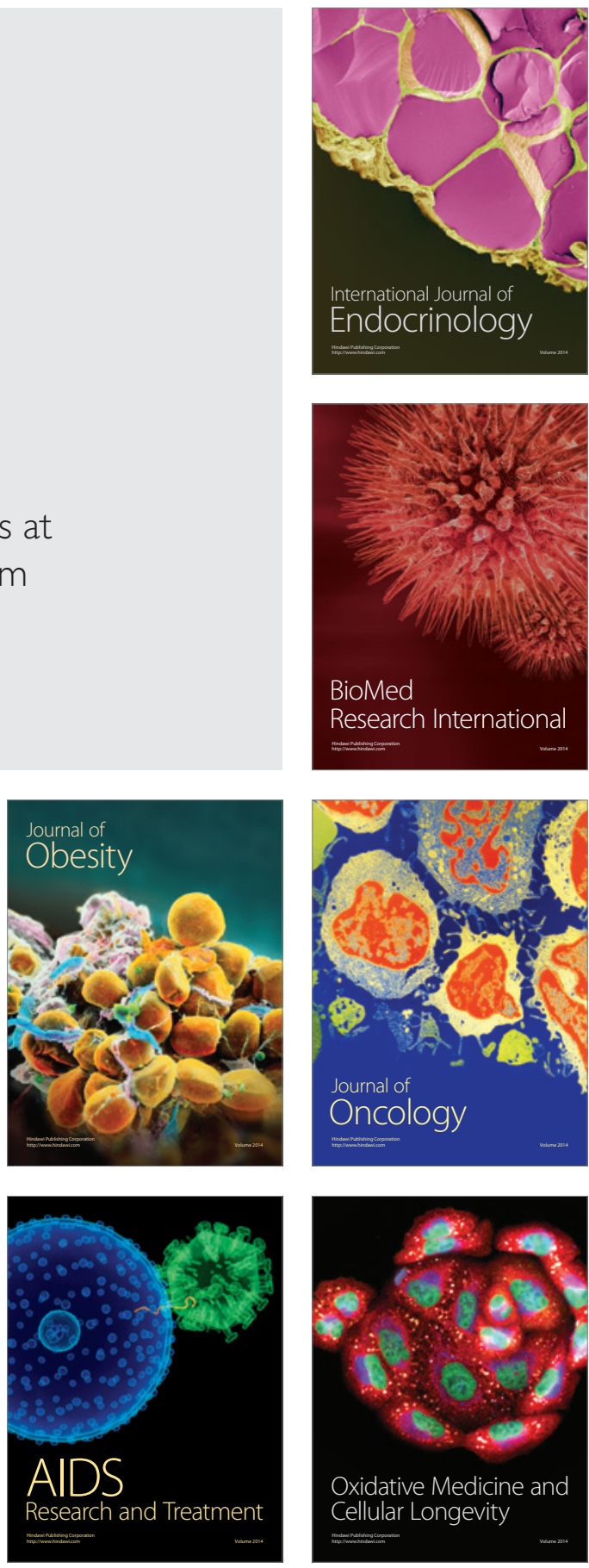